\title{
THE LOCALLY SUPERSYMMETRIC GEOMETRICAL HIERARCHY MODEL
}

\author{
Burt A. OVRUT ${ }^{1}$ \\ Department of Physics, The Rockefeller University, New York, NY 10021, USA \\ and \\ Stuart RABY ${ }^{1}$ \\ Randall Laboratory of Physics, University of Michigan, Ann Arbor, MI 48109, USA
}

Received 18 July 1983

\begin{abstract}
We examine the locally supersymmetric geometrical hierarchy model at energies below the unification mass, $M_{\mathrm{GU}}$. The superfield content, for which the theory has acceptable values of $M_{\mathrm{GU}}$ and $\sin ^{2} \theta_{\mathrm{w}}$, is discussed. We show that radiative corrections induce the spontaneous breakdown of $S U(2) \times U(1)$ to $U(1) E M$ for a top quark mass of $100-200 \mathrm{GeV}$. In theories with two color triplet Higgs superfields a lower bound of $O\left(10^{15}-10^{16} \mathrm{GeV}\right)$ on the supersymmetry breaking parameter, $\mu$, is derived.
\end{abstract}

In a recent paper [1] we extended the geometrical hierarchy model [2] to include $N=1$ supergravity (SUGRY). Our main results were the following.

(A) The value of the potential energy at its absolute minimum (the cosmological constant) can be fine tuned to vanish.

(B) In this case $\mathrm{SU}(5)$ is spontaneously broken, at tree level, to $\mathrm{SU}(3) \times \mathrm{SU}(2) \times \mathrm{U}(1)$. This breaking is a purely supergravitational effect, the scale of which is of $\mathrm{O}(M)$ where $M=M_{\mathrm{Planck}} / \sqrt{8 \pi} \simeq 2.4 \times 10^{18} \mathrm{GeV}$.

(C) SUGRY does not effect supersymmetry (SUSY) breaking. SUSY is spontaneously broken at a scale of $\mathrm{O}\left(\mu^{2}\right)$, where $\mu$ is the dimension 1 parameter in the $O$ 'Raifeartaigh sector of the theory.

(D) We derived the tree level, low energy potential. It divides naturally into two pieces. The first is globally supersymmetric and is independent of supergravitational effects. The second piece explicitly breaks SUSY and is induced by SUGRY. The entire low energy lagrangian is renormalizable.

(E) The parameters of the tree level, low energy potential are such that its absolute minimum occurs at the zero of field space. Furthermore, all scalar

${ }^{1}$ Work support in part by the Department of Energy under Contract Grant Number DE-AC02-81ER40033.B000. masses are of $\mathrm{O}(\mu(\mu / M))$.

We conclude from (E) that, at tree level, SU(2) $X$ $\mathrm{U}(1)$ is unbroken. How, then, is the electroweak group to be broken to $\mathrm{U}(1)_{\mathrm{EM}}$ ? The answer lies in radiative corrections to the potential. These corrections introduce a renormalization point that must be chosen near $M$ for perturbation theory to be valid. It follows that the tree level, low energy potential is only meaningful for field amplitudes of $\mathrm{O}(M)$. To examine the potential energy for small field amplitudes it must be "improved" using the renormalization group (RG). In this paper we carry out the RG improvement of the low energy potential to the oneloop level. We find that $\$ 1$

(a) Higgs fields can have negative (mass) ${ }^{2}$ near the origin of field space and, hence, develop vacuum expectation values (VEV's) that break $\mathrm{SU}(2) \times \mathrm{U}(1) \rightarrow$ $\mathrm{U}(1)_{\mathrm{EM}}$.

(b) Squarks and sleptons, on the other hand, always have non-negative (mass) ${ }^{2}$ near the origin of

$\neq 1$ The breaking of SU(2) $\times \mathrm{U}(1) \rightarrow \mathrm{U}(1) \mathrm{EM}$, through radiative corrections induced by the top quark, has been discussed by the authors of ref. [3]. Our results differ from theirs mainly due to the large threshold effects inherent in the Geometrical Hierarchy Model. 
field space and, hence, have vanishing VEV's. Thus charge, baryon, and lepton numbers remain unbroken .

(c) The size of the electroweak symmetry breaking is set by $\mu(\mu / M)$ and the top quark Yukawa coupling (evaluated near $10^{2} \mathrm{GeV}$ ). Hence, given the electroweak scale, one can calculate the top quark mass. We do this for a wide choice of threshold parameters.

A prerequisite to carrying out these calculations is to evaluate the grand unification mass, $M_{\mathrm{GU}}$, and $\sin ^{2} \theta_{w}$ to the one-loop level. In this paper we evaluate these quantities for a wide choice of threshold parameters. We find that

(d) The values of $M_{\mathrm{GU}}$ and $\sin ^{2} \theta_{\mathrm{w}}$ are, in general, too large. This problem is easily overcome by adding families of Higgs superfields that transform as $\mathbf{1 0}$ and $\overline{10}$ under SU(5). These superfields restore $M_{\mathrm{GU}}$ and $\sin ^{2} \theta_{w}$ to acceptable values.

Finally, we discuss the possibility of reducing, from four to two, the number of Higgs superfields transforming as $\mathbf{5}$ or $\overline{\mathbf{5}}$ under SU(5). To have only two such superfields we find that

(e) scale $\mu$ must be of $\mathrm{O}\left(10^{15}-10^{16} \mathrm{GeV}\right)$, or larger, to sufficiently suppress the amplitude for nucleon decay to kaons + leptons. All calculations will be performed for both two and four Higgs superfields.

The gauge group of our model is $\mathrm{G}=\mathrm{SU}(5)$. The chiral superfields are

(1) A, Z, X. A, Z are 24's and X a 1 under SU(5). These fields form the O'Raifeartaigh sector of the model.

(2) $H, H_{1}, \overline{\mathbf{H}}, \overline{\mathbf{H}}_{1} . \mathrm{H}, \mathrm{H}_{1}$ are 5's and $\overline{\mathrm{H}}, \overline{\mathrm{H}}_{1}$ are 5's under $\mathrm{SU}(5)$. The extra superfields $\mathrm{H}_{1}, \mathrm{H}_{1}$ are introduced to eliminate certain dimension 5 operators which cause the proton to decay too rapidly [2].

(3) $\overline{5}_{J}, 10_{J}, \overline{5}_{J}$ and $10_{J}$ are $\overline{5}$ 's and 10 's respectively under SU(5). We assume there are three leptoquark families $(J=1,3)$.

The superpotential of our model is then given by

$$
\begin{aligned}
W & =\beta \mu^{2} M+\lambda_{1} \mathrm{X}\left(\operatorname{tr} \mathrm{A}^{2}-\mu^{2}\right)+\lambda_{2} \operatorname{tr} Z \mathrm{~A}^{2} \\
& +\lambda_{3} \overline{\mathrm{H}}(\mathrm{A}+m 1) \mathrm{H}_{1}+\lambda_{4} \overline{\mathrm{H}}_{1}(\mathrm{~A}+m 1) \mathrm{H}+\lambda_{I J}^{\mathrm{U}} \mathrm{H} 10_{I} 10_{J} \\
& +\lambda_{I J}^{\mathrm{D}} \overline{\mathrm{H}} 10_{I} 5_{J} .
\end{aligned}
$$

Parameters $\lambda_{i}, \lambda^{\mathrm{U}}, \lambda^{\mathrm{D}}$, and $\beta$ are dimensionless and $\mu$, $m$, and $M$ have dimension 1 . The potential energy, $V$, can be calculated from $W[4]$. To $\mathrm{O}\left(\mu^{3}(\mu / M)\right), V$ has its absolute minimum at

$$
\begin{aligned}
& \langle\mathrm{X}\rangle=\lambda_{2}\left(\lambda_{2}^{2}+30 \lambda_{1}^{2}\right)^{-1 / 2}(\sqrt{3}-1) M, \\
& \langle\mathrm{Z}\rangle=\lambda_{1}\left(\lambda_{2}^{2}+30 \lambda_{1}^{2}\right)^{-1 / 2}(\sqrt{3}-1) M \operatorname{diag}(2,2,2,-3,-3), \\
& \langle\mathrm{A}\rangle=\lambda_{1}\left(\lambda_{2}^{2}+30 \lambda_{1}^{2}\right)^{-1 / 2} \mu \operatorname{diag}(2,2,2,-3,-3)
\end{aligned}
$$

The VEV's of $H, \bar{H}, \overline{5}$, and 10 fields are undetermined at this order. Note that $\langle Z\rangle$ breaks $\mathrm{SU}(5) \rightarrow \mathrm{SU}(3) \times$ $\mathrm{SU}(2) \times \mathrm{U}(1)$ with $M_{\mathrm{GU}}=\lambda_{1}\left(\lambda_{2}^{2}+30 \lambda_{1}^{2}\right)^{-1 / 2}(\sqrt{3}-$ 1) $M$. It follows that, for any choice of $\lambda_{1}$ and $\lambda_{2}$, $M_{\mathrm{GU}} \leqslant[(\sqrt{3}-1) / \sqrt{30}] M \simeq 3.2 \times 10^{17} \mathrm{GeV}$. This vacuum state has vanishing cosmological constant as long as we take

$\beta=-\lambda_{1} \lambda_{2}\left(\lambda_{2}^{2}+30 \lambda_{1}^{2}\right)^{-1 / 2}(2-\sqrt{3})$.

The VEV's of Kahler derivatives $\mathrm{D}_{\phi_{i}} W=\partial \mathrm{W} / \partial \phi_{i}+$ $\left(\phi_{i}^{\dagger} / M^{2}\right) W$, evaluated to $\mathrm{O}\left(\mu^{2}\right)$ at $(2)$, are

$$
\begin{aligned}
& \left\langle\mathrm{D}_{\mathrm{X}} W\right\rangle=-\sqrt{3} \lambda_{1} \lambda_{2}\left(\lambda_{2}^{2}+30 \lambda_{1}^{2}\right)^{-1} \mu^{2}, \\
& \left\langle\mathrm{D}_{\mathrm{Z}} W\right\rangle=-\sqrt{3} \lambda_{1}^{2} \lambda_{2}\left(\lambda_{2}^{2}+30 \lambda_{1}^{2}\right)^{-1} \mu^{2} \operatorname{diag}(2,2,2,-3,-3) .
\end{aligned}
$$

All other $\left\langle\mathrm{D}_{\phi_{i}} W\right\rangle$ vanish to this order. Therefore, SUSY is spontaneously broken by this vacuum state at a scale of $\mathrm{O}\left(\mu^{2}\right)$. Define low energy superpotentials

$$
\begin{aligned}
& \tilde{g}\left(y_{\mathrm{a}},\langle\mathrm{A}\rangle\right)=\mathrm{e}^{(2-\sqrt{3})}\left(\lambda_{3} \overline{\mathrm{H}}(\langle\mathrm{A}\rangle+m 1) \mathrm{H}_{1}\right. \\
& \left.\quad+\lambda_{4} \overline{\mathrm{H}}_{1}(\langle\mathrm{~A}\rangle+m 1) \mathrm{H}+\lambda_{I J}^{\mathrm{U}} \mathrm{H} 10_{I} 10_{J}+\lambda_{I J}^{\mathrm{D}} \overline{\mathrm{H}} 10_{I} \overline{5}_{J}\right)
\end{aligned}
$$

and

$\widetilde{f}\left(Z_{3}, Z_{8}\right)=\left[m_{3 / 2} /(\sqrt{3}-1)\right]\left(\frac{9}{2} \operatorname{tr} Z_{3}^{2}-\frac{4}{3} \operatorname{tr} Z_{8}^{2}\right)$,

where $Z_{3}$ and $Z_{8}$ are the $S U(2)$ triplet and $S U(3)$ octet components of $Z$ respectively, and $m_{3 / 2}$ is the gravitino mass given by

$m_{3 / 2}=\mathrm{e}^{(2-\sqrt{3})} \lambda_{1} \lambda_{2}\left(\lambda_{2}^{2}+30 \lambda_{1}^{2}\right)^{-1 / 2} \mu(\mu / M)$.

Then the tree level, low energy potential is found to be

$V_{\mathrm{LE}}=V_{\mathrm{LE}}^{(1)}\left(y_{\mathrm{a}}\right)+V_{\mathrm{LE}}^{(2)}\left(\mathrm{Z}_{3}, \mathrm{Z}_{8}\right)+\frac{1}{2} \mathrm{D}_{y, \mathrm{Z}, \alpha}^{2}$,

where

$$
\begin{aligned}
& V_{\mathrm{LE}}^{(1)}\left(y_{\mathrm{a}}\right)=\left|\partial \widetilde{g} / \partial y_{\mathrm{a}}\right|^{2}+\left(m_{3 / 2} \tilde{\mathrm{Ag}}+\text { h.c. }\right) \\
& \quad+\left[m_{3 / 2}\left(y_{\mathrm{a}} \partial \widetilde{g} / \partial y_{\mathrm{a}}-\tilde{3 g}\right)+\text { h.c. }\right]+m_{3 / 2}^{2}\left|y_{\mathrm{a}}\right|^{2},
\end{aligned}
$$




$$
\begin{aligned}
& V_{\mathrm{LE}}^{(2)}\left(\mathrm{Z}_{3}, \mathrm{Z}_{8}\right)=\operatorname{tr}\left|\partial \tilde{f} / \partial \mathrm{Z}_{3}\right|^{2}+\operatorname{tr}\left|\partial \tilde{f} / \partial \mathrm{Z}_{8}\right|^{2} \\
& +\left(m_{3 / 2} \mathrm{~A}^{\prime} \tilde{f}+\text { h.c. }\right)+m_{3}^{2}\left(\operatorname{tr}\left|\mathrm{Z}_{3}\right|^{2}+\operatorname{tr}\left|\mathrm{Z}_{8}\right|^{2}\right)
\end{aligned}
$$

and

$A=3-\sqrt{3}, \quad A^{\prime}=-\frac{5}{2}\left(\frac{3}{5} \sqrt{3}-1\right)$,

$\mathrm{D}_{y, Z, \alpha}^{2}$ is the sum of the squares of $\mathrm{SU}(3), \mathrm{SU}(2)$, and $\mathrm{U}(1) \mathrm{D}$ terms. Since the D term associated with U(1) arises from the spontaneous breakdown of SU(5), it contains no dimensional parame ter. Parameter $m$ in (5) is chosen so that the masses of the SU(3) $3(\overline{3})$ components of $\mathrm{H}, \mathrm{H}_{1}\left(\overline{\mathrm{H}}, \overline{\mathrm{H}}_{1}\right)$ are of $\mathrm{O}(\mu)$. Since $|\mathrm{A}|<$ 3 and $\left|A^{\prime}\right|<3$ the absolute minimum of potential (8) occurs at the origin of field space [5]. The masses of all $S U(2)$ doublet Higgs fields, squarks, sleptons, $Z_{3}$, and $Z_{8}$ are of $O\left(m_{3 / 2}\right)$. We will assume that the top quark mass is $\lesssim 10^{2} \mathrm{GeV}$. Parameters $\lambda_{3}, \lambda_{4}$, and $m$ are chosen so that the SU(2) doublet higgsinos have mass between 0 and $10^{2} \mathrm{GeV}$. We use the "minimal" version of SUGRY in which all gaugino masses vanish at tree level. Gauginos are expected to receive masses of $\mathrm{O}\left(\alpha m_{3 / 2}\right)$ from radiative corrections. We will assume these masses are $\$ 10^{2} \mathrm{GeV}$. It follows that there are four mass thresholds in our model.

(1) $10^{2} \mathrm{GeV}$. Particles with masses of $\$ 10^{2} \mathrm{GeV}$ are quarks, leptons, $S U(2)$ doublet higgsinos, gauge fields and gauginos.

(2) $m_{3 / 2}$. Particles with masses of $\mathrm{O}\left(m_{3 / 2}\right)$ are squarks, sleptons, $S U(2)$ doublet Higgs fields, $Z_{3}$ (scalars and fermions) and $Z_{8}$ (scalars and fermions).

(3) $\mu$. Particles with masses of $\mathrm{O}(\mu)$ are SU(3) 3 and $\overline{3}$ Higgs fields and higgsinos.

(4) $M$. Everything else.

The values of $\alpha_{3}$ and $\alpha_{\mathrm{EM}}$ at $10^{2} \mathrm{GeV}$ are chosen to be 10 and $\frac{1}{127}$ respectively. The values of $M_{\mathrm{GU}}$ and $\sin ^{2} \theta_{w}$ (evaluated at $10^{2} \mathrm{GeV}$ ) can be determined from the equations [6]

$$
\begin{aligned}
\alpha_{\mathrm{EM}} & (Q) / \alpha_{3}(Q) \\
\quad & =\frac{3}{8}\left\{1-[\alpha(Q) / \pi]\left[\left(\frac{5}{6} b_{1}+\frac{1}{2} b_{2}-\frac{4}{3} b_{3}\right) \ln \left(M_{\mathrm{GU}} / \mu\right)\right.\right. \\
& +\left(\frac{5}{6} b_{1}^{\prime}+\frac{1}{2} b_{2}^{\prime}-\frac{4}{3} b_{3}^{\prime}\right) \ln \left(\mu / m_{3 / 2}\right) \\
& \left.\left.+\left(\frac{5}{6} b_{1}^{\prime \prime}+\frac{1}{2} b_{2}^{\prime \prime}-\frac{4}{3} b_{3}^{\prime \prime}\right) \ln \left(m_{3 / 2} / Q\right)-\frac{1}{2}\right]\right\}
\end{aligned}
$$

$$
\begin{aligned}
& \sin ^{2} \theta_{\mathrm{w}}(Q)=\frac{3}{8}\left\{1-[5 \alpha(Q) / 6 \pi]\left[\left(b_{1}-b_{2}\right) \ln \left(M_{\mathrm{GU}} / \mu\right)\right.\right. \\
& +\left(b_{1}^{\prime}-b_{2}^{\prime}\right) \ln \left(\mu / m_{3 / 2}\right) \\
& \left.\left.\quad+\left(b_{1}^{\prime \prime}-b_{2}^{\prime \prime}\right) \ln \left(m_{3 / 2} / Q\right)-\frac{1}{3}\right]\right\}
\end{aligned}
$$

where $Q=10^{2} \mathrm{GeV}$. The coefficients $b_{i}$ (threshold dependent) are defined by

$\mathrm{d} \alpha_{i} / \mathrm{d} t=\left(b_{i} / 2 \pi\right) \alpha_{i}^{2}$,

where $\alpha_{3}, \alpha_{2}$, and $\alpha_{1}$ are the running coupling param-

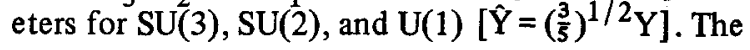
results, for a range of values of $\mu$ and $m_{3 / 2}$, are given in table 1 . In all cases the values of $M_{\mathrm{GU}}$ exceed the bound of $3.2 \times 10^{17} \mathrm{GeV}$ derived earlier. It is, therefore, impossible to adjust parameters $\lambda_{1}$ and $\lambda_{2}$ to account for these values. Furthermore, these unification masses are so large that corrections due to quantum gravitation (incalculable) cannot be ignored. Similarly, in all cases the values of $\sin ^{2} \theta_{w}$ at $10^{2} \mathrm{GeV}$ exceed the experimental upper bound of 0.229 [6]. Finally, for several choices of $\mu$ and $m_{3 / 2}$, the value of $\alpha_{3}$ at $M_{\mathrm{GU}}$ is so large that perturbation theory cannot be trusted. We conclude that the theory described by superpotential (1) does not properly account for $M_{\mathrm{GU}}$ and $\sin ^{2} \theta_{\mathrm{w}}$.

Fortunately, there is a simple solution to this problem [7]. It is clear from eqs. (12) and (13) that both $M_{\mathrm{GU}}$ and $\sin ^{2} \theta_{\mathrm{w}}$ will decrease if one can increase coefficient $b_{1}$ without changing $b_{2}$ and $b_{3}$. Any superfield which transforms as a $\mathbf{1 0}$ under $\mathrm{SU}(5)$ has the $\mathrm{SU}(3) \times \mathrm{SU}(2) \times \mathrm{U}(1)$ decomposition

Table 1

$M_{\mathrm{GU}}, \sin ^{2} \theta_{\mathrm{W}}$, and $\alpha_{3}\left(M_{\mathrm{GU}}\right)$ as functions of $\mu, m_{3 / 2}$, and the number of Higgs doublets, $N_{\mathrm{H}}$.

\begin{tabular}{llllll}
\hline$\mu(\mathrm{GeV})$ & $\begin{array}{l}m_{3 / 2} \\
(\mathrm{GeV})\end{array}$ & $N_{\mathrm{H}}$ & $M_{\mathrm{GU}}(\mathrm{GeV})$ & $\sin ^{2} \theta_{\mathrm{W}}$ & $\alpha_{3}\left(M_{\mathrm{GU}}\right)$ \\
\hline $10^{11}$ & $10^{2}$ & 4 & $7.1 \times 10^{21}$ & 0.245 & 0.48 \\
$10^{11}$ & $10^{3}$ & 4 & $2.9 \times 10^{21}$ & 0.243 & 0.24 \\
$10^{11}$ & $10^{4}$ & 4 & $1.2 \times 10^{21}$ & 0.242 & 0.16 \\
$10^{14}$ & $10^{2}$ & 4 & $7.1 \times 10^{20}$ & 0.256 & 0.20 \\
$10^{14}$ & $10^{4}$ & 4 & $1.2 \times 10^{20}$ & 0.254 & 0.11 \\
$10^{14}$ & $10^{6}$ & 4 & $2.0 \times 10^{19}$ & 0.251 & 0.07 \\
$10^{16}$ & $10^{2}$ & 2 & $3.3 \times 10^{22}$ & 0.237 & 0.13 \\
$10^{16}$ & $10^{4}$ & 2 & $4.2 \times 10^{21}$ & 0.236 & 0.09 \\
$10^{16}$ & $10^{6}$ & 2 & $5.5 \times 10^{20}$ & 0.234 & 0.06 \\
\hline
\end{tabular}


$10=\left(\overline{3}, 1,-\frac{2}{3}\right)+\left(3,2, \frac{1}{6}\right)+(1,1,1)$.

If one can give the $(\overline{3}, 1,-2 / 3)$ and $(3,2,1 / 6)$ components mass of $\mathrm{O}(M)$, and the $(1,1,1)$ component a mass of $\mathrm{O}(\mu)$, then the addition of such a super-

field to the theory will appropriately change the $b_{i}$ coefficients. Henceforth we add $n$ families of 10,10 representations to the theory. For each 10 we must introduce a $\overline{\mathbf{1 0}}$ to make the theory anomaly free. We denote these new superfields as $\widetilde{1}_{i}, \overline{\mathbb{1}}_{i}$ where $i=1$, $n$. To superpotential (1) we add the interaction

$\tilde{\lambda}_{i} \widetilde{\widetilde{10}}_{i}(\mathrm{Z}+\tilde{m} 1) \tilde{10}_{i}$,

where $\widetilde{\lambda}_{i}$ are dimensionless and $\tilde{m}$ has dimension 1 . We chose parameter $\tilde{m}$ in such a way that the SU(3) and SU(2) components of $\langle Z\rangle+\tilde{m} 1$ are of $O(M)$ and $\mathrm{O}(\mu)$ respectively. Parameters $\widetilde{\lambda}_{i}$ and $\widetilde{m}$ can be taken so that $\left\langle\widetilde{10}_{i}\right\rangle=\left\langle\widetilde{10}_{i}\right\rangle=0$ for $i=1, n$. Hence, the $(\overline{3}, 1,-2 / 3),(3,2,1 / 6),(3,1,2 / 3),(\overline{3}, 2,-1 / 6) \mathrm{com}-$ ponent fields get masses of $\mathrm{O}(M)$ and can be ignored. The $(1,1,1),(1,1,-1)$ component fields get masses of $\mathrm{O}(\mu)$ and contribute to the scaling behavior of $\mathrm{U}(1)$ at energies above $\mu$. They both increase coefficient $b_{1}$ and leave $b_{2}, b_{3}$ unchanged. We again calculate $M_{\mathrm{GU}}$ and $\sin ^{2} \theta_{\mathrm{w}}$, this time including the $\widetilde{10}_{i}$, $\widetilde{10}_{i}$ families in the theory. The results, for a range of values of $\mu, m_{3 / 2}$, and $n$, are given in table 2 . In most cases the values of $M_{\mathrm{GU}}$ are below the bound of $3.2 \times 10^{17} \mathrm{GeV}$ derived earlier. It is, therefore, possible to adjust $\lambda_{1}$ and $\lambda_{2}$ to account for these values. Similarly, in most cases the values of $\sin ^{2} \theta_{w}$ at $10^{2}$ $\mathrm{GeV}$ lie between the experimental bound of $0.201-$

Table 2

$M_{\mathrm{GU}}, \sin ^{2} \theta_{\mathrm{W}}$, and $\alpha_{3}\left(M_{\mathrm{GU}}\right)$ as functions of $\mu, m_{3 / 2}, N_{\mathrm{H}}$, and the number of $\widetilde{0}, \overrightarrow{10}$ families, $n$.

\begin{tabular}{lllllll}
\hline$\mu(\mathrm{GeV})$ & $\begin{array}{l}m_{3 / 2} \\
(\mathrm{GeV})\end{array}$ & $N_{\mathrm{H}} n$ & $M_{\mathrm{GU}}(\mathrm{GeV})$ & $\sin ^{2} \theta_{\mathrm{W}}$ & $\alpha_{3}\left(M_{\mathrm{GU}}\right)$ \\
\hline $10^{11}$ & $10^{2}$ & 4 & 4 & $3.2 \times 10^{17}$ & 0.220 & 0.19 \\
$10^{11}$ & $10^{3}$ & 4 & 4 & $1.9 \times 10^{17}$ & 0.219 & 0.14 \\
$10^{11}$ & $10^{4}$ & 4 & 4 & $1.1 \times 10^{17}$ & 0.219 & 0.10 \\
$10^{14}$ & $10^{2}$ & 4 & 6 & $2.7 \times 10^{17}$ & 0.237 & 0.13 \\
$10^{14}$ & $10^{4}$ & 4 & 6 & $1.1 \times 10^{17}$ & 0.236 & 0.08 \\
$10^{14}$ & $10^{6}$ & 4 & 6 & $4.4 \times 10^{16}$ & 0.236 & 0.06 \\
$10^{16}$ & $10^{2}$ & 2 & 15 & $7.2 \times 10^{17}$ & 0.210 & 0.11 \\
$10^{16}$ & $10^{4}$ & 2 & 15 & $4.0 \times 10^{17}$ & 0.213 & 0.08 \\
$10^{16}$ & $10^{6}$ & 2 & 15 & $2.3 \times 10^{17}$ & 0.215 & 0.06 \\
\hline
\end{tabular}

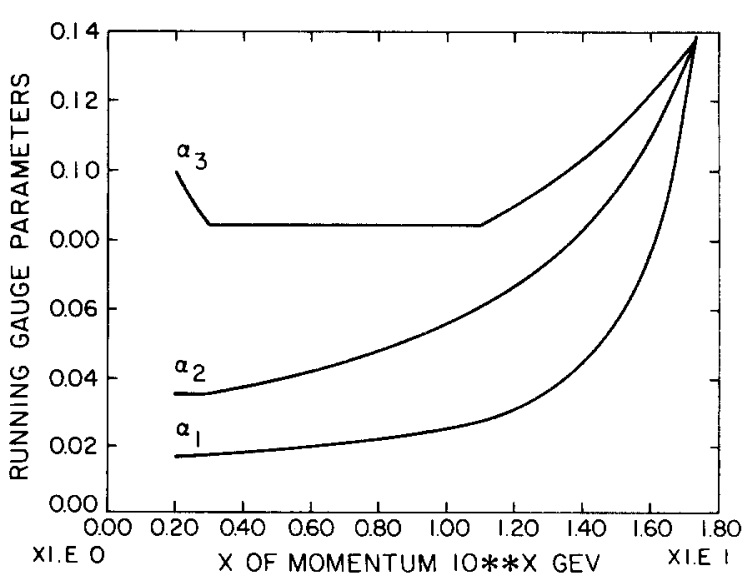

Fig. 1. Running gauge parameters $\alpha_{3}, \alpha_{2}$, and $\alpha_{1}$ as functions of momentum, in a theory with four Higgs doublets, $\mu=10^{11} \mathrm{GeV}, m_{3 / 2}=10^{3} \mathrm{GeV}$, and $n=4$.

0.229. (Recent work indicates that the upper bound might be larger than 0.229 . Hence, all the values for $\sin ^{2} \theta_{w}$ are probably acceptable.) Finally, for all choices of $\mu, m_{3 / 2}$, and $n$, the value of $\alpha_{3}$ at $M_{\mathrm{GU}}$ is sufficiently small that one can trust perturbation theory. We concluse that the modified theory described by superpotential (1), along with interaction (16), properly accounts for $M_{\mathrm{GU}}$ and $\sin ^{2} \theta_{\mathrm{w}}$. A graph of the running gauge coupling parameters for a theory with four Higgs doublets, $\mu=10^{11} \mathrm{GeV}$, $m_{3 / 2}=10^{3} \mathrm{GeV}$, and $n=4$ is shown in fig. 1 . Note the large changes in the $\beta$-functions at each mass threshold. It is these changes that tend to make $M_{\mathrm{GU}}$ and $\alpha_{3}$ large and necessitate the introduction of the $\widetilde{0}_{i}, \widetilde{10}_{i}$ families.

We now consider the question of electroweak symmetry breaking. One can ignore $\mathrm{Z}_{3}, \mathrm{Z}_{8}$ and the SU(3) $3(\overline{3})$ components of $\mathrm{H}, \mathrm{H}_{1}\left(\overline{\mathrm{H}}, \overline{\mathrm{H}}_{1}\right)$ in the low energy lagrangian. The reasons are that (1) the associated superfields do not couple directly to the SU(2) doublet Higgs superfields, (2) $Z_{3}$ and $Z_{8}$ have vanishing hypercharge, and (3) $\mathrm{H}, \mathrm{H}_{1}, \overline{\mathrm{H}}, \overline{\mathrm{H}}_{1}$ have identical tree level mass. Also, it is easy to show that these fields cannot get non-vanishing VEV's from radiative corrections. Hence, they do not contribute to electroweak symmetry breaking to the one-loop level. We absorb the factor $\mathrm{e}^{(2-\sqrt{3})}$ in to the definition of all parameters $\lambda$, and define 
$m_{i}=\lambda_{i}\left[-3 \lambda_{1}\left(\lambda_{2}^{2}+30 \lambda_{1}^{2}\right)^{-1 / 2} \mu+m\right]$,

for $i=3,4$. Then the most general, low energy lagrangian derivable from the geometric hierarchy model is

$$
\begin{aligned}
& \mathcal{L}_{\mathrm{LE}}=\int \mathrm{d}^{2} \theta\left(\hat{m}_{3} \overline{\mathrm{H}}_{1}+\hat{m}_{4} \overline{\mathrm{H}}_{1} \mathrm{H}+\hat{\lambda}_{I J}^{\mathrm{E}} \overline{\mathrm{E}}_{I} \overline{\mathrm{H}} \mathrm{L}_{J}\right. \\
& \left.\quad+\hat{\lambda}_{I J}^{\mathrm{D}} \overline{\mathrm{D}}_{I} \overline{\mathrm{H}} \mathrm{Q}_{J}+\hat{\lambda}_{I J}^{\mathrm{U}} \overline{\mathrm{U}}_{I} \mathrm{HQ}_{J}\right)+\frac{1}{2} \hat{m}_{y_{\mathrm{a}}}^{2}\left|y_{\mathrm{a}}\right|^{2} \\
& \quad+m_{3 / 2}\left(\hat{m}_{3} \hat{\mathrm{C}}_{3} \overline{\mathrm{H}} \mathrm{H}_{1}+\hat{m}_{4} \hat{\mathrm{C}}_{4} \overline{\mathrm{H}}_{1} \mathrm{H}\right) \\
& \quad+m_{3 / 2}\left(\hat{\mathrm{A}}_{I J}^{\mathrm{E}} \hat{\lambda}_{I J}^{\mathrm{E}} \overline{\mathrm{E}}_{I} \overline{\mathrm{H}} \mathrm{L}_{J}+\hat{\mathrm{A}}_{I J}^{\mathrm{D}} \hat{\lambda}_{I J}^{\mathrm{D}} \overline{\mathrm{D}}_{I} \overline{\mathrm{H}} \mathrm{Q}_{J}+\hat{\mathrm{A}}_{I J}^{\mathrm{U}} \hat{\lambda}_{I J}^{\mathrm{U}} \overline{\mathrm{U}}_{I} \mathrm{HQ} Q_{J}\right) \\
& \quad+\text { h.c. }+ \text { kinetic energy terms }
\end{aligned}
$$

where the field in the integrand of $\int d^{2} \theta$ are superfields and all other fields are scalars. $H, H_{1}, \bar{H}, \bar{H}_{1}$ are $\mathrm{SU}(2)$ doublet Higgs fields, $\mathrm{L}_{J}$ and $\mathrm{Q}_{J}$ are $\mathrm{SU}(2)$ doublet left chiral leptons and quarks, and $\overline{\mathrm{E}}_{I}, \overline{\mathrm{U}}_{I}$, and $\overline{\mathrm{D}}_{I}$ are $\mathrm{SU}(2)$ singlet right chiral leptons and quarks. All parameters with "a" run under the renormalization group. For field amplitudes just below $M_{\mathrm{GU}}$ the potential energy associated with (18) becomes identical to (8). Hence, at this scale

$\hat{m}_{i}=m_{i}, \quad \hat{m}_{y_{\mathrm{a}}}=m_{3 / 2}, \quad \hat{\lambda}_{I J}^{\mathrm{E}, \mathrm{D}}=\lambda_{I J}^{\mathrm{D}}, \quad \hat{\lambda}_{I J}^{\mathrm{D}}=\lambda_{I J}^{\mathrm{U}}$,

$\hat{\mathrm{C}}_{3}=\hat{\mathrm{C}}_{4}=\mathrm{A}-1, \quad \hat{\mathrm{A}}_{I J}^{\mathrm{E}}=\hat{\mathrm{A}}_{I J}^{\mathrm{D}}=\hat{\mathrm{A}}_{I J}^{\mathrm{U}}=\mathrm{A}$,

where $A$ is given in (11). We first consider the case where $\hat{m}_{3}=\hat{m}_{4}=0$. The Yukawa coupling parameters of the first two lepto-quark families are sufficiently small that they cannot induce electroweak symmetry breaking. We henceforth consider the third lepto-quark generation only. The potential energy associated with (18) is then given by

$$
\begin{aligned}
& V_{\mathrm{LE}}=\hat{m}_{\mathrm{H}}^{2}|\mathrm{H}|^{2}+\hat{m}_{\mathrm{H}_{1}}^{2}\left|\mathrm{H}_{1}\right|^{2}+\hat{m}_{\mathrm{H}}^{2}|\overline{\mathrm{H}}|^{2}+\hat{m}_{\mathrm{H}_{1}}^{2}\left|\overline{\mathrm{H}}_{1}\right|^{2} \\
& +\hat{m}_{\overline{\mathrm{E}}}^{2}|\overline{\mathrm{E}}|^{2}+\hat{m} \frac{2}{\mathrm{U}}|\overline{\mathrm{U}}|^{2}+\hat{m}_{\overline{\mathrm{D}}}^{2}|\overline{\mathrm{D}}|^{2}+\hat{m}_{\mathrm{L}}^{2}|\mathrm{~L}|^{2}+\hat{m}_{\mathrm{Q}}^{2}|\mathrm{Q}|^{2} \\
& +\left|\hat{\lambda}^{\mathrm{E}} \overline{\mathrm{E}} \mathrm{L}+\hat{\lambda}^{\mathrm{D}} \overline{\mathrm{D}}\right|^{2}+\left|\hat{\lambda}^{\mathrm{U}} \overline{\mathrm{U}}\right|^{2}+\left|\hat{\lambda}^{\mathrm{E}} \overline{\mathrm{H}} \mathrm{L}\right|^{2}+\left|\hat{\lambda}^{\mathrm{D}} \overline{\mathrm{H}} \mathrm{Q}\right|^{2} \\
& +\left|\hat{\lambda}^{\mathrm{U}} \mathrm{HQ}\right|^{2}+\left|\hat{\lambda}^{\mathrm{E}} \overline{\mathrm{E}} \bar{H}^{2}+\right| \hat{\lambda}^{\mathrm{D}} \overline{\mathrm{D}} \overline{\mathrm{H}}+\left.\hat{\lambda}^{\mathrm{U}} \overline{\mathrm{U}}\right|^{2} \\
& +m_{3 / 2}\left(\hat{\mathrm{A}}^{\mathrm{E}} \hat{\lambda}^{\mathrm{E}} \overline{\mathrm{E}} \overline{\mathrm{H}} \mathrm{L}+\hat{\mathrm{A}}^{\mathrm{D}} \hat{\lambda}^{\mathrm{D}} \overline{\mathrm{D}} \overline{\mathrm{H}} \mathrm{Q}+\hat{\mathrm{A}}^{\mathrm{U}} \hat{\lambda}^{\mathrm{U}} \overline{\mathrm{U}} \mathrm{HQ}+\text { h.c. }\right) \\
& +\frac{1}{2} \sum_{i=1}^{3} D_{i}^{2},
\end{aligned}
$$

where

$$
\begin{aligned}
\mathrm{D}_{1} & =-\hat{\mathrm{e}}_{1}\left(\frac{3}{5}\right)^{1 / 2}\left(-\frac{1}{2}|\overline{\mathrm{H}}|^{2}+\frac{1}{2}|\mathrm{H}|^{2}-\frac{1}{2}\left|\overline{\mathrm{H}}_{1}\right|^{2}+\frac{1}{2}\left|\mathrm{H}_{1}\right|^{2}\right. \\
& \left.+|\overline{\mathrm{E}}|^{2}+\frac{1}{3}|\overline{\mathrm{D}}|^{2}-\frac{2}{3}|\overline{\mathrm{U}}|^{2}-\frac{1}{2}|\mathrm{~L}|^{2}+\frac{1}{6}|\mathrm{Q}|^{2}\right) \\
\mathrm{D}_{2}^{\mathrm{a}} & =-\frac{1}{2} \hat{\mathrm{e}}_{2}\left(\overline{\mathrm{H}}^{\dagger} \sigma^{\mathrm{a}} \overline{\mathrm{H}}+\mathrm{H}^{\dagger} \sigma^{\mathrm{a}} \mathrm{H}+\overline{\mathrm{H}}_{1}^{\dagger} \sigma^{\mathrm{a}} \overline{\mathrm{H}}_{1}+\mathrm{H}_{1}^{\dagger} \sigma^{\mathrm{a}} \mathrm{H}_{1}\right. \\
& \left.+\mathrm{L}^{\dagger} \sigma^{\mathrm{a}} \mathrm{L}+\mathrm{Q}^{\dagger} \sigma^{\mathrm{a}} \mathrm{Q}\right)
\end{aligned}
$$

and we have dropped the subscripts indicating the third family. Assume that $\hat{m}_{\mathrm{H}}^{2}<0$, and that all other $\hat{m}_{y_{\mathrm{a}}}^{2}$ are non-negative. Then $V_{\mathrm{LE}}$ has its absolute minimum at

$\langle\mathrm{H}\rangle=\left(\begin{array}{l}0 \\ v\end{array}\right), \quad v=2\left|\hat{m}_{\mathrm{H}}\right|^{2}\left(\hat{\mathrm{e}}_{1}^{2 \frac{3}{5}}+\hat{\mathrm{e}}_{2}^{2}\right)^{-1 / 2}$.

All other VEV's vanish. The $\mathrm{W}$ and $\mathrm{Z}$ boson masses are given by

$m_{\mathrm{W}}^{2}=\frac{1}{2} \hat{\mathrm{e}}_{2}^{2} v^{2}, \quad m_{\mathrm{Z}}^{2}=\frac{1}{2}\left(\hat{\mathrm{e}}_{1}^{2} \frac{3}{5}+\hat{\mathrm{e}}_{2}^{2}\right) v^{2}$.

Eqs. (22) and (23) imply that

$\hat{m}_{\mathrm{H}}^{2}=-\frac{1}{2} m_{\mathrm{Z}}^{2}$.

It follows that $\mathrm{SU}(2) \times \mathrm{U}(1)$ is spontaneously broken to $\mathrm{U}(1)_{\mathrm{EM}}$ if $\hat{m}_{\mathrm{H}}^{2}$, and only $\hat{m}_{\mathrm{H}}^{2}$, becomes negative. To see if this is possible we must discuss the RG equations for the parameters in (20) and (21). The RG equations for $\hat{\alpha}_{1}, \hat{\alpha}_{2}$, and $\hat{\alpha}_{3}$ are given by (14). The RG equations, that are relevant to the value of $\langle\mathrm{H}\rangle$ to the one-loop level, are

$$
\begin{gathered}
\frac{\mathrm{d}}{\mathrm{d} t}\left(\begin{array}{c}
\hat{m}_{\mathrm{H}}^{2} \\
\hat{m}_{\mathrm{U}}^{2} \\
\hat{m}_{\mathrm{Q}}^{2}
\end{array}\right)=\frac{\left(\hat{\lambda}^{\mathrm{U}}\right)^{2}}{8 \pi^{2}}\left(\begin{array}{ccc}
3 & 3 & 3 \\
2 & 2 & 2 \\
1 & 1 & 1
\end{array}\right)\left(\begin{array}{c}
\hat{m}_{\mathrm{H}}^{2} \\
\hat{m}_{\overline{\mathrm{U}}}^{2} \\
\hat{m}_{\mathrm{Q}}^{2}
\end{array}\right) \\
+\frac{\left|\hat{\mathrm{A}}^{\mathrm{U}}\right|^{2}\left(\hat{\lambda}^{\mathrm{U}}\right)^{2} m_{3 / 2}^{2}}{8 \pi^{2}}\left(\begin{array}{l}
3 \\
2 \\
1
\end{array}\right),
\end{gathered}
$$

$\mathrm{d} \hat{\mathrm{A}}^{\mathrm{U}} / \mathrm{d} t=\left[3\left(\hat{\lambda}^{\mathrm{U}}\right)^{2} / 4 \pi^{2}\right] \hat{\mathrm{A}}^{\mathrm{U}}$,

$\mathrm{d} \lambda \mathrm{U} / \mathrm{d} t=3\left(\hat{\lambda}^{\mathrm{U}}\right)^{3} / 8 \pi^{2}-(1 / 2 \pi) \hat{\lambda}^{\mathrm{U}}\left(\frac{8}{3} \hat{\alpha}_{3}+\frac{3}{2} \hat{\alpha}_{2}+\frac{13}{30} \hat{\alpha}_{1}\right)$, where $t=\ln \left(\left|y_{\mathrm{a}}\right| / M_{\mathrm{GU}}\right)$ : Note that $t=0$ when $\left|y_{\mathrm{a}}\right|=$ $M_{\mathrm{GU}}$ and that $t \rightarrow-\infty$ as $\left|y_{\mathrm{a}}\right|$ approaches the origin of field space. The boundary conditions for $\hat{m}_{\mathrm{H}}, \hat{m}_{\mathrm{U}}$, $\hat{m}_{\mathrm{Q}}, \hat{\lambda}^{\mathrm{U}}$, and $\hat{\mathrm{A}}^{\mathrm{U}}$ at $t=0$ are given in (19). The solution to eq. (25) is 
$\hat{m}_{\mathrm{H}}^{2}(t)=\left\{-\frac{1}{2}+\frac{3}{2} \exp \left[-\frac{3}{4 \pi^{2}} \int_{t}^{0} \mathrm{~d} t^{\prime} \hat{\lambda}^{\mathrm{U}}\left(t^{\prime}\right)^{2}\right.\right.$

$$
\left.\times\left(\frac{1+\left|\hat{\mathrm{A}}^{\mathrm{U}}\left(t^{\prime}\right)\right|^{2} m_{3 / 2}^{2}}{\left(2 \hat{m}_{\mathrm{H}}^{2}\left(t^{\prime}\right)+m_{3 / 2}^{2}\right)}\right)\right] m_{3 / 2}^{2},
$$

$\hat{m}_{\tilde{U}}^{2}(t)=\frac{2}{3} \hat{m}_{\mathrm{H}}^{2}(t)+\frac{1}{3} m_{3 / 2}^{2}$,

$\hat{m}_{\mathrm{Q}}^{2}(t)=\frac{1}{3} \hat{m}_{\mathrm{H}}^{2}(t)+\frac{2}{3} m_{3 / 2}^{2}$.

At $t=0, \hat{m}_{\mathrm{H}}^{2}(0)=\left(-\frac{1}{2}+\frac{3}{2}\right) m_{3 / 2}^{2}=m_{3 / 2}^{2}$, the correct boundary condition. For $t \rightarrow \frac{3}{\infty}_{\infty}, \hat{m}_{\mathrm{H}}^{2}(t) \rightarrow\left(-\frac{1}{2}+\right.$ 0) $m_{3 / 2}^{2}=-\frac{1}{2} m_{3 / 2}^{2}$, so that $\hat{m}_{\mathrm{H}}^{2}$ can indeed become negative. Similarly, $\hat{m}_{\mathrm{U}}^{2}(0)=\hat{m}_{\mathrm{Q}}^{2}(0)=m_{3 / 2}^{2}$, the correct boundary conditions. For $t \rightarrow-\infty, \hat{m}_{\mathrm{U}}^{2}(t) \rightarrow 0$ and $\hat{m}_{\mathrm{Q}}^{2}(t) \rightarrow \frac{1}{2} m_{3 / 2}^{2}$, so that these parameters are always non-negative. It follows that squark VEV's vanish and, hence, baryon number and charge remain unbroken. The solution to eq. (26) is

$\hat{\mathrm{A}}^{\mathrm{U}}(t)=(3-\sqrt{3}) \exp \left(-\frac{3}{4 \pi^{2}} \int_{t}^{0} \mathrm{~d} t^{\prime} \hat{\lambda}^{\mathrm{U}}\left(t^{\prime}\right)^{2}\right)$.

It follows that the value for $\hat{\mathrm{A}}^{\mathrm{U}}$ decreases as $t \rightarrow-\infty$.

Eq. (27) is most easily solved numerically. However, it is clear that $\frac{1}{3}\left(\frac{8}{3} \hat{\alpha}_{3}+\frac{3}{2} \hat{\alpha}_{2}+\frac{13}{30} \hat{\alpha}_{1}\right)$ acts as an ultraviolet (UV) attractive fixed point for $\hat{\alpha}^{U}=\hat{\lambda}^{U 2} / 4$.

Finally, the RG equations for the remaining masses are

$\mathrm{d} \hat{m}_{y}^{2} / \mathrm{d} t=0$.

Hence, $\hat{m}_{\mathrm{H}_{1}}^{2}(t)=\hat{m}_{\mathrm{H}}^{2}(t)=\hat{m}_{\overline{\mathrm{H}}_{1}}^{2}(t)=\hat{m}_{\overline{\mathrm{E}}}^{2}(t)=\hat{m}_{\overline{\mathrm{D}}}^{2}(t)=$ $\hat{m}_{\mathrm{L}}^{2}(t)=m_{3 / 2}^{2}>0$. Therefore no Higgs field, other than $\mathrm{H}$, has a non -vanishing VEV. Furthermore, all slepton VEV's vanish and lepton number and charge remain unbroken. It follows that $\hat{m}_{\mathrm{H}}^{2}$, and only $\hat{m}_{\mathrm{H}}^{2}$, can be non-negative. From eqs. (22), (27), (28), and (31) we see that the size of the electroweak symmetry breaking, $v$, is determined by $m_{3 / 2}$ and the top quark Yukawa coupling, $\hat{\lambda}^{\mathrm{U}}$, evaluated at $10^{2} \mathrm{GeV}$. Therefore, given $v$ and $m_{3 / 2}$, one can evaluate the top quark mass using the equation

$m_{\text {top }}=\hat{\lambda}^{\mathrm{U}}\left(10^{2} \mathrm{GeV}\right) v$,

VEV $v$ is related to $m_{Z}$ by eq. (23). Henceforth, take $m_{\mathrm{Z}}=93.8 \mathrm{GeV}$ [6]. Using eqs. (12), (14) and (23) we can determine $v$. The results for $v, \hat{\alpha} \mathrm{U}$ at $10^{2} \mathrm{GeV}$, and $m_{\text {top }}$, for a range of values of $\mu, m_{3 / 2}$, and $n$, are given in table 3 , Parameter $\hat{\alpha}^{\mathrm{U}}$ is driven toward $\mathrm{UV}$
Table 3

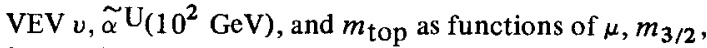
$N_{\mathrm{H}}$, and $n$.

\begin{tabular}{lllllll}
$\mu(\mathrm{GeV})$ & $\begin{array}{l}m_{3 / 2} \\
(\mathrm{GeV})\end{array}$ & $N_{\mathrm{H}}$ & $n$ & $v(\mathrm{GeV})$ & $\begin{array}{l}\hat{\alpha} \mathrm{U}\left(10^{2}\right. \\
\mathrm{GeV})\end{array}$ & $\begin{array}{l}m_{\text {top }} \\
(\mathrm{GeV})\end{array}$ \\
\hline $10^{11}$ & $10^{2}$ & 4 & 4 & 174.67 & 0.109 & 204.84 \\
$10^{11}$ & $10^{3}$ & 4 & 4 & 173.82 & 0.058 & 148.61 \\
$10^{11}$ & $10^{4}$ & 4 & 4 & 173.99 & 0.054 & 143.46 \\
$10^{14}$ & $10^{2}$ & 4 & 6 & 179.24 & 0.107 & 208.13 \\
$10^{14}$ & $10^{4}$ & 4 & 6 & 179.86 & 0.053 & 146.61 \\
$10^{14}$ & $10^{6}$ & 4 & 6 & 180.04 & 0.049 & 141.35 \\
$10^{16}$ & $10^{2}$ & 2 & 15 & 171.77 & 0.108 & 200.29 \\
$10^{16}$ & $10^{4}$ & 2 & 15 & 172.93 & 0.053 & 141.19 \\
$10^{16}$ & $10^{6}$ & 2 & 15 & 174.53 & 0.049 & 136.83 \\
\hline
\end{tabular}

attractive fixed point $\frac{1}{3}\left(\frac{8}{3} \hat{\alpha}_{3}+\frac{3}{2} \hat{\alpha}_{2}+\frac{13}{30} \hat{\alpha}_{1}\right)>\frac{8}{9} \hat{\alpha}_{3}=$ 0.089 at $10^{2} \mathrm{GeV}$. It follows that $\hat{\alpha}^{\mathrm{U}}$ at $10^{2} \mathrm{GeV}$ and the top quark mass are relatively large. In all cases, however, $\hat{\alpha}^{\mathrm{U}}$ is sufficiently small that one can trust perturbation theory. A graph of $m_{\text {top }}$ as a function of $m_{3 / 2}$, for four Higgs doublets, $\mu=10^{11} \mathrm{GeV}$, and $n=4$, is shown in fig. 2 . We conclude that when $\hat{m}_{3}=$ $\hat{m}_{4}=0, \mathrm{H}$, and only $\mathrm{H}$, can get a non-vanishing VEV. This VEV breaks $\mathrm{SU}(2) \times \mathrm{U}(1) \rightarrow \mathrm{U}(1)_{\mathrm{EM}}$ at the correct scale as long as the top quark is sufficiently massive. VEV $\langle\mathrm{H}\rangle$ gives mass to "up" quarks only. Therefore, when $\hat{m}_{3} \rightarrow \hat{m}_{4}=0$, "down" quarks and leptons remain massless.

In order to give masses to "down" quarks and leptons, $\mathrm{H}$ must develop a non-vanishing VEV. In a

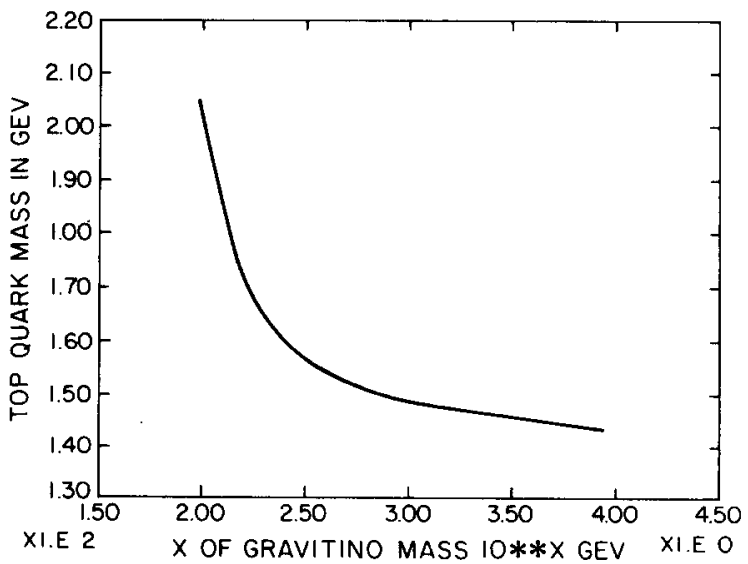

Fig. 2. The top quark mass as a function of $m_{3 / 2}$, in a theory with four Higgs doublets, $\mu=10^{11} \mathrm{GeV}$, and $n=4$. 
theory with four Higgs doublets this can only occur beyond the one-loop level in perturbation theory, or if we consider "non-minimal" versions of SUGRY. These possibilities will be explored elsewhere. Consider a theory with two Higgs doublets $\left(\hat{m}_{3}, \hat{m}_{4}\right.$ are replaced by $\hat{m}$, and $\hat{\mathrm{C}}_{3}, \hat{\mathrm{C}}_{4}$ are replaced by $\hat{\mathrm{C}}$, in (18)). Assume $\hat{m} \neq 0$. Then, from the potential energy associated with (18), we find that

$\langle\overline{\mathrm{H}}\rangle=\left(\begin{array}{l}\widetilde{v} \\ 0\end{array}\right), \quad \widetilde{v}=-m_{3 / 2} v \hat{m} \hat{\mathrm{C}} /\left(\hat{m}_{\mathrm{H}}^{2}-\left|\hat{m}_{\mathrm{H}}^{2}\right|\right)$.

The RG equations associated with $\hat{m}$ and $\hat{\mathrm{C}}$ are

$\mathrm{d} \hat{m} / \mathrm{d} t=(\hat{m} / 4 \pi)\left(3 \hat{\alpha}^{\mathrm{U}}-\hat{\alpha}_{1}-3 \hat{\alpha}_{2}\right)$,

$\mathrm{d} \hat{\mathrm{C}} / \mathrm{d} t=-3 \hat{\alpha}^{\mathrm{U}} / 2 \pi-\hat{\mathrm{A}}^{\mathrm{U}}$,

where the boundary condition for $\hat{\mathrm{C}}$ at $t=0$ is given in (19). The solutions to eqs. (35) and (36) are

$$
\begin{aligned}
\hat{m}(t) & =\hat{m}(0) \exp \left(-\frac{1}{4 \pi} \int_{t}^{0} \mathrm{~d} t^{\prime}\right. \\
\times & {\left.\left[3 \hat{\alpha}^{\mathrm{U}}\left(t^{\prime}\right)-\hat{\alpha}_{1}\left(t^{\prime}\right)-3 \hat{\alpha}_{2}\left(t^{\prime}\right)\right]\right) }
\end{aligned}
$$

and

$$
\hat{\mathrm{C}}(t)=2-\sqrt{3}+\int_{t}^{0} \mathrm{~d} t^{\prime}\left[(3 / 2 \pi) \hat{\alpha}^{\mathrm{U}}\left(t^{\prime}\right)+\hat{\mathrm{A}}^{\mathrm{U}}\left(t^{\prime}\right)\right],
$$

respectively. If one chooses $\hat{m}(0) \ll m_{3 / 2}$, our previous results will not be substantially altered. Henceforth, we take $\hat{m}(0)=10^{-2} m_{3 / 2}$, which satisfies this criterion. Using our previous results, and eqs. (34), (37), and (38), we can determine $\widetilde{v}$. The results for $\widetilde{v}, \tilde{m}$ at $10^{2} \mathrm{GeV}$, and $\hat{\mathrm{C}}$ at $10^{2} \mathrm{GeV}$, for a range of values of $m_{3 / 2}$, are given in table 4 . We conclude that in a theory with two Higgs doublets, $\overline{\mathrm{H}}$ can de-

Table 4

VEV $\tilde{v}, \hat{m}\left(10^{2} \mathrm{GeV}\right), \hat{\mathrm{C}}\left(10^{2} \mathrm{GeV}\right)$ as functions of $m_{3 / 2}$ and $\hat{m}\left(M_{\mathrm{GU}}\right)$, in a theory where $\mu=10^{16} \mathrm{GeV}, N_{\mathrm{H}}=2$, and $n=$ 15.

\begin{tabular}{lllll}
\hline$m_{3 / 2}$ & $\begin{array}{l}m\left(M_{\mathrm{GU}}\right) \\
(\mathrm{GeV})\end{array}$ & $\tilde{v}(\mathrm{GeV})$ & $\hat{m}\left(10^{2} \mathrm{GeV}\right)$ & $\hat{\mathrm{C}}\left(10^{2} \mathrm{GeV}\right)$ \\
\hline $10^{2}$ & 1 & -64.429 & $9.367 \times 10^{-1}$ & 22.454 \\
$10^{4}$ & $10^{2}$ & -85.289 & $1.346 \times 10^{2}$ & 36.626 \\
$10^{6}$ & $10^{4}$ & -76.467 & $1.251 \times 10^{4}$ & 35.023 \\
\hline
\end{tabular}

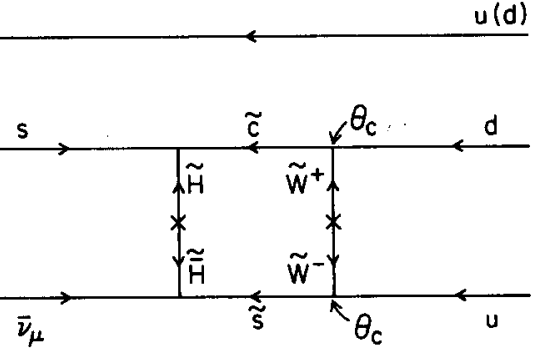

Fig. 3. The main graph contributing to nucleon decay modes $\mathrm{p}(\mathrm{n}) \rightarrow \mathrm{K}^{+}\left(\mathrm{K}^{0}\right) \bar{\nu}_{\mu}$.

velop a non-vanishing VEV that is sufficiently large to give perturbative masses to all "down" quarks and leptons.

Finally, we want to consider the implications on proton decay of reducing the number of 5 and $\overline{5}$ Higgs superfields from four to two. In theories with four Higgs superfields the decay of nucleons into bosons and leptons is naturally suppressed [2]. In theories with two Higgs superfields this is no longer the case. The main graph contributing to the dominant decay modes, $\mathrm{p}(\mathrm{n}) \rightarrow \mathrm{K}^{+}\left(\mathrm{K}^{0}\right) \bar{\nu}_{\mu}$, is shown in fig. 3. The decay amplitude associated with this graph is [8]

$\mathrm{A} \propto b_{0} g_{\text {cuu }}$,

where

$$
\begin{aligned}
& b_{0}=\left(G_{\mathrm{F}} / \sqrt{2}\right)\left(m_{\mathrm{W}}^{2} / 32 \pi^{2}\right) m_{\tilde{\mathrm{W}}} \\
& \quad \times\left[f\left(m_{\tilde{\mathrm{q}}}, m_{\tilde{\mathrm{q}}}, m_{\tilde{\mathrm{W}}}\right)+f\left(m_{\tilde{\mathrm{q}}}, m_{\tilde{\mathrm{\ell}}}, m_{\tilde{\mathrm{W}}}\right)\right], \\
& f\left(m_{1}, m_{2}, m_{3}\right)=\left(m_{2}^{2}-m_{3}^{2}\right)^{-1} \\
& \quad \times\left[m_{2}^{2}\left(m_{1}^{2}-m_{2}^{2}\right)^{-1} \ln \left(m_{1}^{2} / m_{2}^{2}\right)\right. \\
&\left.\quad-m_{3}^{2}\left(m_{1}^{2}-m_{3}^{2}\right)^{-1} \ln \left(m_{1}^{2} / m_{3}^{2}\right)\right]
\end{aligned}
$$

and

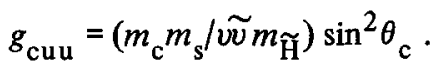

The " " denotes the superpartner to a standard particle. $\widetilde{\mathrm{H}}$ and $\widetilde{\mathrm{H}}$ are the higgsinos associated with the color triplet components of Higgs scalars in $\mathrm{H}$ and $\overline{\mathrm{H}}$. One expects that

$m_{\widetilde{\mathrm{w}}}\left[f\left(m_{\tilde{\mathrm{q}}}, m_{\tilde{\mathrm{q}}}, m_{\tilde{\mathrm{w}}}\right)+f\left(m_{\tilde{\mathrm{q}}}, m_{\tilde{\mathrm{\ell}}}, m_{\tilde{\mathrm{w}}}\right) \simeq 1 / m_{\mathrm{w}}\right.$ 
Define $\bar{b}_{0}$ by

$\bar{b}_{0}=\left(G_{\mathrm{F}} / \sqrt{2}\right) m_{\mathrm{W}} / 32 \pi^{2}=2.2 \times 10^{6} \mathrm{GeV}^{-1}$.

From (39) (with $b_{0} \approx \bar{b}_{0}$ ), (44), and the present lower bound on the nucleon lifetime $\left(\tau_{n} \gtrsim 3 \times 10^{30}\right.$ $y r)$, one can infer that

$g_{\text {cuu }}<10^{-7} / M$.

Therefore,

$b_{0} g_{\text {cuu }} \simeq \bar{b}_{0} g_{\text {cuu }}<\bar{b}_{0} 10^{-7} / M$,

which, using (42), implies that

$\left(b_{0} / \bar{b}_{0}\right)\left[\left(m_{\mathrm{c}} m_{\mathrm{s}} / v \widetilde{v}\right) \sin ^{2} \theta_{\mathrm{c}}\right] 10^{7} M<m_{\widetilde{\mathrm{H}}}$.

Assuming that $m_{\tilde{\mathrm{q}}}=m_{\tilde{\mathrm{l}}}=\bar{m}$ (a good approximation)

$b_{0} / \bar{b}_{0}=m_{\mathrm{W}} / \bar{m}, \quad$ for $\bar{m}=m_{\widetilde{\mathrm{W}}}$,

$$
=2 m_{\widetilde{\mathrm{W}}} m_{\mathrm{W}} / \bar{m}^{2}, \quad \text { for } \bar{m} \gg m_{\widetilde{\mathrm{W}}},
$$$$
=\left(2 m_{\mathrm{W}} / m_{\widetilde{\mathrm{W}}}\right) \ln \left(m_{\widetilde{\mathrm{W}}}^{2} / \bar{m}^{2}\right), \text { for } \bar{m} \ll m_{\widetilde{\mathrm{W}}} \text {. }
$$

Clearly, the smallest value of $b_{0} / \bar{b}_{0}$ is obtained when $\bar{m} \gg m_{\widetilde{w}}$. Taking typical values for $m_{\mathrm{W}}=10^{2} \mathrm{GeV}$, $m_{\mathrm{c}}=1.5 \mathrm{GeV}, m_{\mathrm{s}}=0.15 \mathrm{GeV}, \sin \theta_{\mathrm{c}}=0.2$, and $v=$ $\widetilde{v}=250 / \sqrt{2} \mathrm{GeV}$, we find that

$m_{\widetilde{\mathrm{H}}}>2 \times 10^{15} \mathrm{GeV}\left(m_{\widetilde{\mathrm{W}}} / 2 \mathrm{GeV}\right)\left(10^{6} \mathrm{GeV}^{2} / \bar{m}^{2}\right)$.

The tree level expression for $m_{\widetilde{H}}$ (in terms of $\mu$ ) can be derived from (1) and (2). We conclude that, in order for our theory to have only two $5, \overline{5}$ Higgs doublets and still be consistent with the lower bound on the nucleon lifetime, it is necessary for

$$
\begin{aligned}
\mu & >\left(1 / 6.83 \lambda_{3}\right)\left(M / M_{\mathrm{GU}}\right)\left(2 \times 10^{15} \mathrm{GeV}\right) \\
& \times\left(m_{\widetilde{\mathrm{W}}} / 2 \mathrm{GeV}\right)\left(10^{16} \mathrm{GeV}^{2} / \bar{m}^{2}\right) .
\end{aligned}
$$

For typical parameters (50) implies that $\mu>\mathrm{O}\left(10^{15}\right.$ $10^{16} \mathrm{GeV}$ ).

We would like to thank J. Hagelin for useful conversations.

\section{References}

[1] B. Ovrut and S. Raby, Phys. Lett. B125 (1983) 270.

[2] S. Dimopoulos and S. Raby, Los Alamos preprint LAUR-82-1982 (1982);

R. Barbieri, S. Ferrara and D.V. Nanopoulos, Z. Phys. C13 (1982) 276, CERN TH 3305 (1982);

M. Dine and W. Fischler, Nucl. Phys. B204 (1982) 346;

J. Polchinski and L. Susskind, Phys. Rev. D26 (1982)

3661 ;

T. Banks and V. Kaplunovsky, Nucl. Phys. B206 (1982) 45.

[3] L. Alverez-Gaume, M. Claudson and M. Wise, Nucl. Phys. B207 (1982) 16;

L. Ibanez, Madrid preprint FTUAM/82-8 (1982);

J. Ellis, D. Nanopoulos and K. Tamvakis, CERN preprint TH-3418 (1982);

L. Alverez-Gaume, J. Polchinski and M. Wise, Harvard preprint HUTP82/A063;

H. Nilles, Phys. Lett. 115B (1982) 193 ;

K. Inoue, A. Kakuto, H. Komatsu and S. Takeshita, Prog. Theor. Phys. 68 (1982) 927.

[4] E. Cremmer, S. Ferrara, L. Girardello and T. van Proeyen, CERN preprint TH-3348 (1982);

J. Bagger and E. Witten, Phys. Lett. B118 (1982) 103.

[5] H. Nilles, M. Srednicki and D. Wyler, Phys. Lett. 124B (1983) 337.

[6] See M.A.B. Beg and A. Sirlin, Phys. Rep. 88 (1982), and references therein.

[7] A. Masiero, D. Nanopoulos, K. Tamvakis and T. Yanagida, Phys. Lett. 115B (1982) 299.

[8] J. Ellis, J. Hagelin, D. Nanopoulos and K. Tamvakis, Phys. Lett. 124B (1983) 484. 